\title{
Los instrumentos jurídicos ante la discriminación remunerativa entre mujeres y hombres en Chile
}

\author{
Irene Rojas Miño*
}

\section{RESUMEN}

A partir del reconocimiento del principio de igualdad, en particular el de la remuneración entre mujeres y hombres y de las obligaciones internacionales que el Estado de Chile ha contraído al efecto, en este artículo se pretende dar cuenta del estado actual en Chile de los instrumentos jurídicos que operan frente a esta específica discriminación, y de las limitaciones que ellos plantean para la eficacia del principio de igual remuneración por trabajo de igual valor entre mujeres y bombres.

Mujer - discriminación - remuneración

\section{Legal instruments in the face of remuneration discrimination between women and men in Chile}

\begin{abstract}
Based on the recognition of the principle of equality, in particular that of remuneration between women and men, and the international obligations that the Chilean State has contracted to this purpose, this article intends to account for the current status in Chile of the legal instruments that operate presence of this specific discrimination, and the limitations they present to the effectiveness of the principle of equal remuneration for equal value work between women and men.
\end{abstract}

Woman - discrimination - remuneration

* Licenciada en Ciencias Jurídicas y Sociales, Universidad de Chile. Doctora en Derecho, Universidad de Valencia, España. Profesora de Derecho del Trabajo, Universidad de Talca, Chile. Correo electrónico: irojas@utalca.cl

Este artículo forma parte del Proyecto de Investigación Fondecyt Regular No 1191000, "Definición de los instrumentos que garanticen la igualdad de remuneraciones entre mujeres y hombres".

Artículo recibido el 14.1.2020 y aceptado para su publicación el 18.6.2020. 


\section{INTRODUCCIÓN}

$\mathrm{E}$ 1 principio de igualdad está reconocido internacionalmente y, a la vez, por los diversos Estados, lo que significa la prohibición universal de la discriminación. Ciertamente que el principio de igualdad comprende el ámbito de las relaciones laborales y, entre sus múltiples dimensiones, el derecho de igual remuneración por trabajo de igual valor.

Sin embargo, un dato cierto es el de la existencia de la brecha salarial -entendiendo por tal "la diferencia entre los ingresos medios de hombres y mujeres como porcentaje de los ingresos de los hombres" 1 -, la que se plantea en los diversos países ${ }^{2}$, y que en el caso de Chile ello se reitera ${ }^{3}$; así al año 2018 tal brecha medida en ingresos medios alcanza al $27,2 \%{ }^{4}$. Aunque este concepto debe ser observado, en cuanto se presentan diversas formas de medir tal diferencia; no obstante, todas las cifras muestran una diferencia en perjuicio de la mujer ${ }^{5}$.

En todo caso, es cierto que la brecha salarial plantea dos componentes: una "parte explicada", fundamentada principalmente en los supuestos "atributos del mercado del trabajo" 6 , y una "parte no explicada", que corresponde a las diferencias de remuneración sin fundamento alguno, es decir, la parte discriminatoria ${ }^{7}$. No obstante, estas categorías deben relativizarse, en cuanto algunos atributos del mercado del trabajo pueden constituir un supuesto discriminatorio al encubrir menores remuneraciones a las labores desarrolladas principalmente por mujeres, en razón de estereotipos que desvalorizan sus trabajos. Además, como ha planteado un sector de la doctrina laboralista, la brecha salarial en su conjunto no es sino la manifestación más evidente de la discriminación de la mujer en el mundo del trabajo, y su superación, por consiguiente, requiere de "políticas antidiscriminatorias generales, globales y transversales..."

Sin embargo, este trabajo limita el análisis de los instrumentos jurídicos ante la discriminación remunerativa en el sistema chileno ${ }^{9}$, considerando en todo caso tanto la discriminación manifiesta como la encubierta.

${ }^{1}$ Organización Internacional del Trabajo, OIT, 2013, p. 14.

${ }^{2}$ OIT, 2019, pp. 19-48.

${ }^{3}$ Véase por todos, Comunidad Mujer, 2015, pp. 1-12.

${ }^{4}$ Instituto Nacional de Estadísticas, INE, 2019, p. 4.

${ }^{5}$ El índice más utilizado es la comparación basada en la hora de trabajo, sin embargo, el tema es mucho más complejo. Al respecto, véase OIT, 2019, pp. 22-30.

${ }^{6}$ OIT, 2019, pp. 19-21.

${ }^{7}$ La que también se reitera en el caso chileno. Así, un estudio efectuado a base de la Encuesta Casen de 2013 plantea que esta componente significaría "un salario 17,2\% menor que el de los hombres solo por el hecho de ser mujer" (Brega et al., 2015, p. 27).

${ }^{8}$ Ballester, 2018a, pp. 10-11.

${ }^{9}$ Esto, a causa de la necesaria delimitación del objeto de estudio, aunque coincido plenamente con la doctrina que sostiene que la brecha salarial no es sino la manifestación de la discriminación de la mujer en el mundo del trabajo. 
Para este fin, el punto de partida lo constituye el derecho de igual remuneración entre mujeres y hombres por trabajo de igual valor, considerando, en primer lugar, el reconocimiento de este derecho por parte de la comunidad internacional, así como lo plantean diversos tratados internacionales, algunos de estos han sido ratificados por el Estado de Chile, y, en segundo lugar, la obligación de los Estados de definir los instrumentos que garanticen efectivamente este derecho, como lo establecen los mismos tratados internacionales.

A partir de dicha fundamentación, este trabajo plantea los objetivos de evaluar el nivel de cumplimiento de dichos compromisos internacionales, junto con identificar los instrumentos jurídicos existentes en el sistema chileno frente a esta específica discriminación y, además, las limitaciones que ellos presentan en la finalidad de garantizar eficazmente el principio de igual remuneración por trabajo de igual valor entre mujeres y hombres.

En definitiva, de una parte, se analizan la fundamentación jurídica del derecho de no discriminación y el contenido del principio de igual remuneración por trabajo de igual valor y, de la otra, se identifican y analizan los instrumentos jurídicos que existen en el sistema jurídico de relaciones laborales chileno relacionados con la discriminación salarial. Además, se presenta un apartado final de conclusiones.

I. EL DERECHO DE NO DISCRIMINACIÓN REMUNERATIVA EN RAZÓN DEL GÉNERO: SU FUNDAMENTACIÓN, SU CONTENIDO Y SU EFICACIA

\section{Su fundamentación}

Del derecho de igual remuneración por trabajo de igual valor. Si bien el derecho de no discriminación remunerativa se fundamenta en el principio general de igualdad ante la ley, debe recordarse que no será hasta el siglo XX que se reconozca la plena igualdad de hombres y mujeres, "tanto ante la ley -o en la aplicación de la ley-como en la ley -o en el contenido de la ley-" 10 . Precisamente, será la igualdad ante la ley el fundamento teórico de la prohibición de trato diferenciado, configurándose así el principio de no discriminación y, en el marco del ámbito laboral, el de igual remuneración por trabajo de igual valor.

Al efecto, el principio de igualdad y el consiguiente derecho de no discriminación han sido reconocidos como derechos fundamentales en los diversos tratados internacionales, así como se plantea en la Declaración Universal de Derechos Humanos, de 1948, la que también reconoce expresamente el derecho la igualdad de salario pero lo limita al "trabajo igual" (Artículo 23.2). No será hasta 1970 que Naciones Unidas, mediante el Pacto de Derechos Económicos Sociales y Culturales, reconozca en el derecho a un salario equitativo e igual por trabajo de igual valor (Artículo 7.a.i).

\footnotetext{
${ }^{10}$ LousAdA, 2014, p. 41.
} 
Sin embargo, han sido instrumentos internacionales específicos los que han explicitado el principio de igual remuneración al incorporar la trascendental referencia al trabajo de igual valor entre mujeres y hombres, como lo hace la Convención sobre la Eliminación de Todas las Formas de Discriminación contra la Mujer (CEDAW, por su sigla en inglés), de Naciones Unidas (1979), la que lo incorporó en la sección laboral: "El derecho a igual remuneración, inclusive prestaciones, y a igualdad de trato con respecto a un trabajo de igual valor, así como a igualdad de trato con respecto a la evaluación de la calidad del trabajo" (Artículo 11, letra d). Se debe resaltar que tal convención dio origen a un órgano de seguimiento y control, el CEDAW (Comité para la Eliminación de la Discriminación contra la Mujer), del que ha emanado una importante doctrina acerca de la aplicación de este principio.

Desde el Derecho Internacional del Trabajo, la Organización Internacional del Trabajo (OIT) ha reconocido el principio general de igualdad en materia laboral, como se plantea en general en el Convenio 111 (1958), relativo a la discriminación en materia de empleo y ocupación. Aunque acerca del derecho de no discriminación retributiva es más preciso el Convenio No 100 (1951), precisamente referido "a la igualdad de remuneración por trabajo de igual valor”. Es este último texto normativo el que establece la obligación de “... garantizar la aplicación a todos los trabajadores del principio de igualdad de remuneración entre la mano de obra masculina y la mano de obra femenina por un trabajo de igual valor" (Artículo 2.1).

Además, tratados internacionales de ámbito regional han reconocido expresamente el derecho de igual remuneración por trabajo de igual valor, como sucede en Europa con la Carta Social Europea de $1961^{11}$. Con posterioridad, la Unión Europea ha sido fuente de una extensa normativa que reconoce y desarrolla la garantía de igual retribución entre mujeres y hombres, junto con establecer las garantías para su efectividad ${ }^{12}$, normativa que también se manifiesta en la doctrina emanada del Tribunal de Justicia de la Unión Europea (en adelante, TJUE). En la actualidad está vigente la Directiva 2006/54, referida a la Igualdad de Oportunidades e Igualdad de Trato entre Hombres y Mujeres, la que dedica el Capítulo 1 a la igualdad de retribución, requiriendo a los Estados la efectividad de los instrumentos de desarrollo de tales mandatos. Asimismo, en la garantía de estos derechos es de particular importancia la Recomendación de la Comisión de 7 de marzo de 2014, la que, junto con insistir en la eficacia de tales garantías, reclama la necesaria transparencia de las remuneraciones.

En la perspectiva de las normas internacionales de carácter regional en el ámbito latinoamericano, es admisible hacer notar la falta de un expreso reconocimiento del derecho de no discriminación entre hombres y mujeres y de retribución por trabajo de igual valor. Por consiguiente, en este ámbito las fuentes internacionales directas del

${ }^{11}$ La que expresamente reconoce en su artículo 4, 3. “...el derecho de los trabajadores de ambos sexos a una remuneración igual por un trabajo de igual valor” (ver Consejo de Europa).

12 De la materia, véase, entre otros, BALlester, 2018b, CABEZAs, 2012 (pp. 79-98) y Lousada, 2014. 
derecho de igual remuneración por trabajo de igual valor las constituyen los convenios de la OIT, en especial el número 100, y la CEDAW, de la ONU.

\section{Su contenido}

Como se ha señalado, en una perspectiva universal el principio de igualdad prohíbe la discriminación de remuneración por trabajo de igual valor entre mujeres y hombres. Por consiguiente, son tres los conceptos que incorpora y que deben ser analizados a este respecto: el de remuneración, el de no discriminación y el de trabajo de igual valor.

(a) En primer lugar, el concepto de comparación en este derecho es el de "remuneración" -o de "retribución”, como se lo denomina en sistemas jurídicos específicos-, el que corresponde a todas las contraprestaciones por el trabajo y, como señala la OIT, "comprende el salario o sueldo ordinario, básico o mínimo, y cualquier otro emolumento en dinero o en especie pagados por el empleador, directa o indirectamente, al trabajador, en concepto del empleo de este último" (Art. 1 a). Por consiguiente, se abandona el criterio anterior que limitaba la comparación a la remuneración base, como es el salario.

(b) En segundo lugar, el derecho a la no discriminación es el derecho de igualdad de trato. Así, desde el Derecho Internacional la discriminación plantea "una diferencia de trato que no es razonable ni objetiva" ${ }^{3}$ y, desde la perspectiva del Derecho del trabajo, "toda exclusión o menoscabo en el trabajo, fundado en criterios distintos de la capacidad o idoneidad del trabajador..." 14 . La particularidad laboral de la discriminación remunerativa de género es que, además de su masividad en los distintos sistemas de relaciones laborales, presenta diversas manifestaciones.

En todo caso, estas expresiones de discriminación remunerativa se enmarcan en categorías generales que ya habían sido definidas por la disciplina del Derecho Antidiscriminatorio ${ }^{15}$, así como son las de discriminación directa e indirecta ${ }^{16}$. La discriminación es directa si se efectúa a base del criterio que define a la persona que es discriminada, mientras que la discriminación es indirecta cuando se usa un criterio no definitorio que, sin embargo, tiene el efecto de desfavorecer al grupo en cuestión ${ }^{17}$. Así, la vulneración al principio de igualdad de remuneración comprende tanto la discriminación directa como indirecta, como lo establecen las normas internacionales aplicables, especialmente el CEDAW y el Convenio 100 de la OIT.

A su vez, respecto de las dos categorías que se presentan en la discriminación remunerativa, se plantean al interior de cada una de ellas diversas manifestaciones, todas

13 Díaz, 2013, pp. 637-638.

${ }^{14}$ CaAmaño, 2003, pp. 25-26.

${ }^{15}$ Como señala MuÑoz (2018, p. 176), el Derecho Antidiscriminatorio se ha “... constituido plenamente como una rama relativamente específica del saber jurídico y de la práctica jurisprudencial...”.

${ }^{16}$ BARrere, 1996 y SÁEz, 1994.

${ }^{17}$ Si bien el concepto de "discriminación indirecta" nace en el Derecho Antidiscriminatorio de Estados Unidos, el mismo ha sido desarrollado en el Derecho Europeo, particularmente por el TJUE, a partir de pronunciamientos acerca de discriminación de mujeres contratadas a tiempo parcial (CAAMAÑO, 2001, pp. 67-81). 
necesarias a considerar al momento de definir los instrumentos jurídicos para la restauración del principio de igualdad.

La discriminación directa plantea remuneraciones distintas en razón del sexo, aun cuando se realice el mismo trabajo, por lo que el criterio de diferenciación es el sexo del trabajador: una mayor cuantía si es hombre, un menor monto si es mujer.

Si bien es claro el alcance general de esta categoría de discriminación, las manifestaciones que plantea son diversas. Estas son: primero, la discriminación de remuneración por trabajos iguales y que tienen la misma denominación; al respecto, se ha sostenido en sistemas comparados que este supuesto es el de menor envergadura por su evidente vulneración jurídica ${ }^{18}$. Segundo, la discriminación de remuneración por trabajos iguales a los que se les otorga diversa denominación según cuál sea el sexo de quien los ejecute, y, "normalmente, los trabajos designados con nombres femeninos están peor pagados que los designados con nombres masculinos" 19 . Y, tercero, la discriminación remunerativa con ocasión de la maternidad, la que plantea dos supuestos: uno de ellos es la discriminación con ocasión del ejercicio de los derechos de maternidad, particularmente el de permisos y descansos ${ }^{20}$; y, si bien esta materia se relaciona en parte con la seguridad social, es necesario efectuar el análisis desde la perspectiva de la verificación de un tratamiento diverso. El otro supuesto es el de la "penalización" de las trabajadoras por ser madres ${ }^{21}$, que muestra una brecha salarial entre las que son y las que no son madres.

La discriminación indirecta, por su parte, es la que afecta a un colectivo de personas, en este caso las trabajadoras, a base de la aplicación de un criterio supuestamente neutral. Esta discriminación incorpora dos supuestos: (a) El primero es la infravaloración de los trabajos realizados mayoritariamente por mujeres, en cuanto se muestra una segregación horizontal de las mujeres en el trabajo, que confina a un gran número de las mujeres a determinados sectores de producción y servicios y, a la vez, a actividades o profesiones específicas ${ }^{22}$. (b) El segundo es la menor remuneración por exclusión de determinadas formas de retribución a puestos de trabajos que atienden a alguna modalidad especial de contratación, en circunstancias de que dichas modalidades son utilizadas principalmente por mujeres, como es el contrato a jornada parcial.

Debe advertirse que los diversos estudios constatan la prevalencia de discriminación indirecta, en cuanto devalúa el trabajo que desempeñan mayoritariamente las mujeres ${ }^{23}$ y que así se mantiene en la actualidad ${ }^{24}$. Además, en los distintos casos de discriminación se debe atender a los mecanismos requeridos para identificarla y definirla, para ello se acude a la comparación, pero no solo de trabajos similares, sino que también a base de

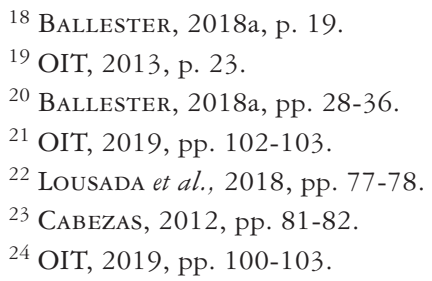


un comparador flexible o un comparador hipotético, como lo ha establecido el Derecho Comunitario $^{25}$.

(c) En tercer lugar, la explicitación del derecho de no discriminación por "trabajo de igual valor" responde a una de las manifestaciones de discriminación indirecta: los trabajos feminizados han sido y son evaluados en razón de estereotipos negativos, lo que lleva a otorgarles una consideración social inferior y, por ello, una remuneración menor ${ }^{26}$.

Entonces, se requiere revalorizar el trabajo; es decir, definir su valía para determinar su remuneración ${ }^{27}$ pero ahora a base de criterios objetivos, como señalan los diversos instrumentos internacionales ${ }^{28}$. Esta exigencia ya había sido establecida por el Convenio 100 de la OIT, el que en su artículo 3 prescribió la adopción de “... medidas para promover la evaluación objetiva del empleo tomando como base los trabajos que este entrañe...”. Precisamente, la OIT ha generado una guía acerca de la promoción de la igualdad salarial, la que incorpora métodos para "comparar trabajos y determinar el valor igual”29.

\section{Su eficacia (o la necesaria adopción de medidas que garanticen el derecho de igualdad en las remuneraciones)}

El derecho de igual remuneración por trabajo de igual valor no se garantiza con el solo reconocimiento jurídico. En efecto, la discriminación que se verifica tiene raíces en una cultura global que minusvalora a la mujer y, en este caso, a las trabajadoras, reproduciéndose condiciones inequitativas en el mundo del trabajo.

Es por ello la necesidad de establecer los instrumentos necesarios, tanto jurídicos como de políticas públicas, con el fin de garantizar condiciones de equidad en el trabajo. En el ámbito remunerativo han sido los mismos tratados internacionales los que establecen tal obligación respecto de los Estados. Así, el CEDAW plantea el compromiso de los Estados de "adoptar las medidas adecuadas para eliminar las discriminaciones que afectan a la mujer en el empleo" y asegurar el derecho de igual remuneración respecto de un trabajo de igual valor (Art. 11, letra d). La OIT, por su parte, establece en el Convenio 100 la obligación de cada Estado de "promover (...) y garantizar (...) la aplicación a todos los trabajadores del principio de igualdad de remuneración entre la mano de obra masculina y la mano de obra femenina por un trabajo de igual valor" (Artículo 2.1); además, la Resolución del Consejo de Administración de la OIT del 2004 convocó a los respectivos gobiernos a la contribución activa para que eliminen las diferencias de remuneración basadas en el género (Artículo 1.v.).

\footnotetext{
${ }^{25}$ Así, la Directiva 2006/54 de la Unión Europea.

${ }^{26}$ LOUSADA et al., 2018, p. 88.

27 OIT, 2013, p. 42.

${ }^{28}$ En este sentido, es clara la Recomendación del 2014 de la UE: "El valor del trabajo deberá evaluarse y compararse utilizando criterios objetivos, tales como requisitos educativos, profesionales y de formación, la cualificación, el esfuerzo y la responsabilidad, los trabajos realizados y la naturaleza de las tareas en cuestión".

${ }^{29}$ OIT, 2013, pp. 41-53.
} 
A los efectos de la promoción establecida, se presentan diversos instrumentos. En una perspectiva de aproximación, en razón de los que han definido e implementado los sistemas comparados ${ }^{30}$, se pueden establecer las siguientes categorías de instrumentos:

En primer lugar, el establecimiento de los conceptos requeridos en la configuración de la discriminación, de la igualdad de remuneración y, particularmente, de trabajo de igual valor, junto con las vías administrativas y judiciales encargadas de conocer los reclamos y acciones por vulneraciones al principio de igualdad.

En segundo lugar, la definición de "modelos proactivos" 31 que se establecen en el sistema de relaciones laborales para el logro del objetivo indicado. Es importante señalar que estos modelos son diversos, definiéndose en razón de las particularidades de cada sistema; sin embargo, no es inocua la adopción de uno u otro mecanismo. Al efecto, se plantea un estudio comparativo entre diversos modelos ${ }^{32}$, en el que uno impone la obligación a los empleadores de efectuar el ejercicio de equidad remunerativa (Canadá y Suecia), otro es de fomento de la equidad mediante la participación voluntaria de los empleadores (Reino Unido y Países Bajos) y el último plantea instrumentos normativos por medio de la ley (Francia y Suiza), concluyendo que el que ha obtenido mejores resultados es el primero.

En tercer lugar, el establecimiento de entidades que colaboren con la implementación de las políticas definidas y, a la vez, permitan el seguimiento de estas políticas, como sucede en Canadá y Suecia.

No obstante, las medidas efectuadas hasta ahora son insuficientes para superar la discriminación remunerativa, la que se mantiene en diversos sistemas. A partir de ello se definen a nivel internacional tanto instrumentos generales ${ }^{33}$ o específicos, como es la necesaria "transparencia salarial”, como lo establece la Recomendación de la Comisión de la Unión Europea del 2014³4, o la necesaria evaluación no sexista de los empleos que formula la OIT a efectos de asistencia técnica a los mismos Estados ${ }^{35}$.

\section{Los instrumentos Vigentes EN Chile y SU EFICACiA}

\section{El derecho de no discriminación}

En el sistema jurídico chileno diversas fuentes normativas reconocen expresamente el principio de igualdad y, consiguientemente, el derecho de no discriminación. Así,

\footnotetext{
${ }^{30}$ Para este fin, se efectuó un estudio preliminar de la normativa en sistemas de Derecho Comparado, el que consultó los diversos países que han definido políticas públicas en materia de discriminación salarial: "Gesetz zur Auskunft über Gehalt" (Alemania, 2017), "Employment Equity Act" (Canadá, 1998), "Employment Equality Act” (Irlanda, 1998), "Equality Act” (Reino Unido, 2010) y “Discrimination Act” (Suecia, 2008).

${ }^{31}$ En la denominación utilizada por Chicha y OIT, 2006.

32 Chicha y OIT, 2006, pp. 8-27.

${ }^{33}$ Entre otros documentos, la Directiva Comunitaria 2006/54 referida a “... la aplicación de igualdad de oportunidades e igualdad de trato entre hombres y mujeres en asuntos de empleo y ocupación”.

${ }^{34}$ Unión Europea, 2014.

35 OIT, 2008, pp. 1-6.
} 
la Constitución Política vigente reconoce la igualdad de todas las personas como base institucional, garantizándose "la igualdad ante la ley" (Art. 19, n. 2 inciso 1.ro), por lo que ni la ley ni autoridad alguna podrán establecer diferencias arbitrarias (Art. 19, n. 2 inciso 2.do). En el ámbito laboral de las garantías constitucionales, "se prohíbe cualquier discriminación que no se base en la capacidad o idoneidad personal..."36 (Art. 19, n. 16, inciso 3.ro), prohibición que se ha interpretado como manifestación del principio general de igualdad ${ }^{37}$.

La ley laboral, por su parte, ha desarrollado el contenido de este derecho en el ámbito de las relaciones de trabajo, toda vez que el Código del Trabajo (en adelante, CT) reconoce que "son contrarios a las leyes laborales los actos de discriminación". Pero un mecanismo idóneo de protección solo se estableció mediante la acción de tutela de derechos fundamentales del trabajo en el 2008, la que si bien limitó los derechos fundamentales amparados, el de no discriminación sí está cubierto por esta acción, aunque con alguna exclusión ${ }^{38}$. De otra parte, la misma ley laboral ha reconocido dos derechos específicos de no discriminación de sexo, estos son por embarazo para acceder, permanecer o promover un puesto de trabajo (Artículo 194, inciso final, CT) ${ }^{39}$ y el de igual remuneración por igual trabajo (Artículo 62 bis del CT).

En este contexto, el derecho de igual remuneración por trabajo de igual valor debería entenderse incorporado al ordenamiento jurídico, toda vez que el Estado de Chile ha ratificado los dos tratados universales que expresamente lo reconocen: el Convenio 100 de la OIT y la CEDAW, de ONU; sin embargo, este reconocimiento no se reitera en una fuente interna.

Precisamente, la interrogante se plantea respecto del reconocimiento y garantías en el sistema jurídico chileno del derecho de no discriminación remunerativa por trabajo de igual valor, para ello debe distinguirse entre el específico instrumento establecido para garantizar este derecho y las demás vías que comprende el ordenamiento jurídico para la tutela de los derechos laborales.

En todo caso, el concepto jurídico de remuneración sí da cuenta de los diversos elementos que la integran (Art. $41 \mathrm{CT}$ ), lo que coincide con la exigencia establecida por OIT.

\section{La limitada garantía del "derecho de igualdad de remuneraciones", Ley $N^{\circ} 20.348$}

En el 2009 se dictó y entró en vigencia la Ley N 20.348, la que "resguarda el derecho a la igualdad de las remuneraciones”, para esto estableció la obligación general

${ }^{36}$ Aunque se agrega que ello es ... sin perjuicio de que la ley pueda exigir la nacionalidad chilena o límites de edad para determinados casos.

37 CaAmaño, 2003, p. 29.

38 Como prescribe el inciso segundo del Art. 485 del CT: También se aplicará este procedimiento para conocer de los actos discriminatorios a que se refiere el artículo $2^{\circ}$ de este Código, con excepción de los contemplados en su inciso sexto. Estos últimos son los referidos a trato diferenciado por obligaciones financieras.

${ }^{39}$ Ley $\mathrm{N}^{\circ} 19.591$, de 1998. 
del empleador a “...dar cumplimiento al principio de igualdad de remuneraciones entre hombres y mujeres que presten el mismo trabajo”.

Junto con el establecimiento de este derecho laboral se estableció una regulación para su ejercicio y garantía: (a) Un derecho a reclamo ante la misma empresa, la que debe establecer un procedimiento al efecto. (b) Se condicionó la acción judicial por lesión a derechos fundamentales al reclamo previo ante la empresa. (c) Se estableció la obligación de las grandes empresas (de 200 o más trabajadores) de establecer un registro que "consigne los diversos cargos o funciones en la empresa y sus características técnicas esenciales". (d) Se definió un incentivo para el cumplimiento de no discriminación salarial mediante la rebaja de multas de otras infracciones laborales; sin embargo, dicho estímulo se cambió por una posterior modificación legal ${ }^{40}$. (e) Finalmente, se estableció una obligación similar en un área de la administración pública, respecto de los trabajadores a contrata.

Ciertamente que el reconocimiento del derecho de igualdad fue limitado -el derecho se restringió a la igual remuneración por igual trabajo, para ello se estableció un derecho a reclamo al interior de las empresas, vía que condicionó incluso la acción judicial de tutela del derecho, además de definirse un registro de los puestos de trabajo pero solo en la gran empresa- y la interrogante que se plantea es por qué el reconocimiento de este derecho se dio en estos términos.

Junto con considerar la tardanza en esta modificación legal, la que se explica por el tardío reconocimiento en Chile de la vigencia de los derechos fundamentales en las relaciones laborales ${ }^{41}$, como también del establecimiento de instrumentos ante la discriminación de género, lo cierto es que podría estimarse que la propuesta inicial del reconocimiento expreso de este derecho y de sus garantías ya era limitada, pero ella habría podido tener algún impacto en el sistema de relaciones laborales. Sin embargo, en la discusión al interior del Congreso Nacional se efectuó un vaciamiento de su contenido.

El origen del Proyecto de Ley fue una Moción presentada por un grupo de diputa$\operatorname{dos}^{42}$, la que, fundada en el principio de igualdad y en el derecho de no discriminación, planteó establecer "un dispositivo jurídico de protección ante la discriminación de remuneración”, para lo que se hace una propuesta bastante escueta referida a su establecimiento explícito, planteándose incorporar como norma laboral el que en materia de remuneraciones debería aplicarse el principio de igualdad por un servicio de igual valor. Para ello se estableció un instrumento específico: el establecimiento de un registro de cargo en toda empresa con más de cinco trabajadores, que consignara los diversos cargos o funciones ejercidos por sus trabajadores, designando el número de personas que los desempeñan y señalando los nombres de quienes actualmente los ejercen. Este registro sería público, en cuanto estaría a disposición de cualquiera de sus trabajadores y del inspector del trabajo. Sin embargo, en la discusión legislativa de este Proyecto se incorporaron drásticas

\footnotetext{
${ }^{40}$ Ley $\mathrm{N}^{\circ} 20.416$, de 2010, que sustituyó dicho criterio.

${ }^{41}$ Lo que ocurre únicamente a fines de la década de los noventa del siglo XX.

${ }^{42}$ BCN, 2009, pp. 3-7.
} 
modificaciones, particularmente las que tuvieron su origen en el Senado, por lo que debe distinguirse entre ambas cámaras ${ }^{43}$.

En la discusión que se planteó en su Cámara de origen (de Diputados) se adoptaron medidas específicas, algunas de estas relativizaron el alcance de las modificaciones ${ }^{44}$. Pero fue en el Senado, específicamente en la Comisión de Trabajo, donde se incorporaron las radicales modificaciones a la propuesta planteada: (a) La limitación de la garantía a trabajos iguales en vez de trabajos de igual valor. (b) La exclusión de la garantía en razón de las diferencias objetivas, capacidades, calificaciones, idoneidad, responsabilidad o productividad. (c) El aumento del umbral de la obligación de registro de los puestos de trabajos solo a la gran empresa, es decir de 200 y más trabajadores. (d) El condicionamiento de la acción de tutela de derechos fundamentales al reclamo previo dentro de la empresa. (e) La limitación de la garantía en la administración pública a los cargos de contrata.

Para entender los motivos para tal modificación de la propuesta inicial, es necesario analizar el contenido de la discusión en la Comisión de Trabajo del Senado.

(a) Respecto de la restricción de la garantía a trabajos iguales en vez de trabajos de igual valor, fue a partir de un cuestionamiento al objeto del derecho de igualdad ("un servicio de igual valor") y de la presión de cambiarlo por "un trabajo de igual productividad” que se acordó limitar su contenido a la no discriminación en la remuneración por igual trabajo. En efecto, por dudas acerca del concepto de "servicio de igual valor" 45 , como también de su contenido, alcance y cuál entidad sería la que decidiría por tal materia ${ }^{46}$, y porque además dichas interrogantes fueron en parte respondidas por la presentación del Servicio Nacional de la Mujer (SERNAM) ${ }^{47}$, dos senadores proponen limitar tal principio "al trabajo de igual productividad" 48 . Frente a ello, el Ejecutivo plantea una nueva Indicación en que elimina el trabajo por igual valor y lo sujeta a igual trabajo ${ }^{49}$, propuesta que es aprobada.

(b) En cuanto a la exclusión de la garantía a "las diferencias de remuneración basadas en capacidades, calificaciones, idoneidad, responsabilidad o productividad", esta fue nuevamente una solución de consenso frente a las objeciones que entonces se plantearon

${ }^{43}$ El Poder Legislativo en Chile plantea dos Cámaras: la de Diputados y la de Senadores, y la tramitación de una ley contempla su discusión en ambas.

44 Véase Oficio de Cámara de Origen a Cámara Revisora, de 10 de abril de 2008, Biblioteca del Congreso Nacional, BCN, 2009, pp. 89-90: (a) se identificó al titular de la obligación: el empleador; (b) se aumentó el umbral de las empresas que tendrían tal obligación (solo las de más de cincuenta trabajadores), todo ello con el fundamento de que tal exigencia "podría entrabar el funcionamiento de las micro y pequeñas empresas"; (c) se definió la procedencia del reclamo ante la misma empresa; (d) se estableció el incentivo para las empresas por cumplimiento de tales normas, y (e) se incorporó al sector de la administración pública la misma obligación de igual remuneración por trabajo de igual valor.

45 Así lo plantea el senador Pizarro (BCN, 2009, p. 103).

46 Como lo planteó la entonces senadora Matthei (BCN, 2009, p. 102).

${ }^{47}$ El actual Ministerio de la Mujer y Equidad de Género, al 2008 era solo un servicio, el SERNAM.

${ }^{48}$ Estos fueron los senadores Allamand y Pérez Varela (BCN, 2009, p. 115).

49 BCN, 2009, p. 122. 
a la propuesta de la Cámara de Diputados. Debido a esto se propuso que existirían “... distinciones en función de otras circunstancias objetivas y válidas, tales como la capacidad, la calificación, la responsabilidad o el rendimiento de las personas" 50 . Si bien se discutió y objetó tal premisa en razón de que la consagración legal de la prohibición de discriminación ya consideraba como tal "la exclusión o preferencia definida en las calificaciones” (artículo 4, inciso 5.to, CT), se aprobó una nueva indicación que planteó la directora del SERNAM por la que se incorporan tales criterios ${ }^{51}$.

(c) El aumento del umbral de la obligación de registro de los puestos de trabajo solo a la gran empresa, por su parte, tuvo su origen en una indicación cuyo fundamento fue el que la mediana empresa no podría cumplir tal exigencia o que ello constituía una gran dificultad ${ }^{52}$, en cuanto que “... las empresas cambian su estructura y organización constantemente, porque están en permanente movimiento, con lo cual llevar un registro (...) es una labor engorrosa..." 53 . Si bien tal argumento fue reiterado, no fue objeto de grandes objeciones, en circunstancias de que se está rechazando la base del sistema de clasificación profesional y de puestos de trabajo, los que sí existen en la mediana y gran empresa y que están institucionalizados en diversos sistemas comparados e, inclusive, remitidos su definición por medio de la autonomía colectiva.

Otro argumento que se planteó para rechazar el registro de los cargos y funciones fue la dificultad de establecerlos por la incidencia en ellos de los cambios estructurales de las empresas, "por cuanto difícilmente podrá llevarse al día la descripción de tales funciones, toda vez que estas se encuentran en constante evolución" 54 . Ciertamente que en este argumento se expone una confusión entre la organización estructural de las empresas y las actividades que ella realiza, en cuanto si bien hay una relación ello en caso alguno impide tal definición de los puestos de trabajo.

Un tercer argumento de rechazo fue el que la multiplicidad de funciones que realizan o deben realizar los trabajadores, a causa de la polifuncionalidad impuesta por las empresas -lo que está autorizado en el sistema chileno- ${ }^{55}$, dificulta la confección del registro y encarece o hace más compleja la gestión de la empresa ${ }^{56}$.

Si bien los representantes del SERNAM y también un senador de la Comisión rechazaron tal aumento del umbral de posición, en la Cámara de Trabajo del Senado fue aprobada por mayoría dicha indicación ${ }^{57}$.

(d) El condicionamiento de la acción de tutela de derechos fundamentales al reclamo previo dentro de la empresa se estableció al interior de la Comisión de Trabajo del Senado, la que, en el marco de la próxima entrada en vigencia de la acción de tutela de

\footnotetext{
${ }^{50}$ Como lo planteó el senador Allamand (BCN, 2009, p. 126).

${ }^{51}$ BCN, 2009, p. 131.

52 BCN, 2009, p. 133.

53 BCN, 2009, p. 133.

54 Planteado por la entonces senadora Matthei (BCN, 2009, p. 135).

55 Incorporada por la Ley $\mathrm{N}^{\circ} 19.759$, de 2001, en artículo 10. n. 3, CT.

56 Senador Pizarro (BCN, 2009, pp. 135-136).

57 Por 3 votos contra 2 .
} 
derechos laborales del ámbito laboral ${ }^{58}$, planteó el condicionamiento de esta acción al reclamo previo dentro de la empresa ${ }^{59}$, propuesta que fue aceptada de inmediato y se adopta como acuerdo de dicha Comisión.

(e) Por último, se limitó la garantía en la administración pública a los cargos de contrata, en el entendido de que esa fue la premisa sostenida en la Cámara de Diputados ${ }^{60}$. Asimismo, de igual manera que la garantía general, se elimina la referencia al trabajo de igual valor y se incorporó la referencia al grado y la calificación.

La totalidad de estas modificaciones fueron acogidas sin mayor discusión en la Sala del Senado, salvo la referida a la limitación de las empresas obligadas a establecer el registro de los puestos de trabajo, materia que fue llevada a votación y se impuso la propuesta de la Comisión de Trabajo ${ }^{61}$.

Si bien posteriormente, en la Cámara de origen de este proyecto, fueron objetadas varias de las modificaciones introducidas, y catalogadas en algunos casos como "escollo a la igualdad de género", estas modificaciones fueron aceptadas con el fin de evitar una Comisión Mixta que las volviera a ver, debido a un temor de mayor retroceso ${ }^{62}$.

\section{La nula eficacia de la Ley $N^{\circ} 20.348$}

Esta ley entró en vigencia en junio de 2009 y sus preceptos más relevantes fueron incorporados como artículo 62 bis del CT. Sin embargo, rápidamente fueron señaladas sus deficiencias y finalmente su ineficacia frente al desafío de garantizar la igualdad de remuneraciones entre mujeres y hombres.

Así, los dos organismos internacionales respecto de los que el Estado de Chile tenía compromisos en la materia plantearon las serias deficiencias de esta ley. Por su parte, el CEDAW, en el Informe del 2012, planteó su "preocupación” entre otras materias por la exclusión del “... principio de igualdad de remuneración por un trabajo de igual valor” y recomendó al Estado de Chile, que “... considere la posibilidad de modificar su legislación para incluir el principio de igualdad de remuneración por un trabajo de igual valor" 63 .

Un reproche similar planteó la OIT en un Informe de la Comisión de Expertos ${ }^{64}$, el que, además de cuestionar la explicación del gobierno de Chile frente a la sola

${ }^{58}$ Esto es en el 2008.

59 Fue el asesor legislativo del Ministerio del Trabajo quien planteó dicha propuesta (BCN, 2009 p. 139). Esto no deja de llamar la atención, porque el gobierno de entonces era uno de posiciones políticas de centroizquierda, por lo que podría haberse analizado el efecto perverso de este condicionamiento de la acción judicial.

${ }^{60}$ Así lo plantea la Historia de la Ley N 20.348 al interior del debate de la Comisión del Trabajo en la Cámara (BCN, 2009, p. 59).

${ }^{61}$ En efecto, se aprobó la propuesta de la Comisión del Trabajo en esta materia por 16 votos contra 9 (BCN, 2009, p. 159).

${ }^{62}$ Así lo plantea la diputada Saa (BCN, 2009, p. 168).

${ }^{63}$ CEDAW. 2012, pp. 8 y 9.

${ }^{64}$ OIT, 2012 , pp. 536 y 537. 
garantía de no discriminación para igual trabajo ${ }^{65}$, señala: “... la Comisión estima que el sistema establecido contribuye a la persistencia de la brecha de remuneración y de la segregación ocupacional por motivo de sexo..."66, por lo que solicita la revisión del citado Art. 62 bis del CT.

Más drástico fue el Informe elaborado al interior de la Cámara de Diputados en "Evaluación de la Ley N²0.348”67. Tras un profundo análisis, este Informe concluyó en la ineficacia de la normativa establecida por dicha ley, particularmente por la dificultad de efectuar las denuncias, toda vez que no es posible obtener información acerca de las remuneraciones de sus compañeros y, además, por el temor a ser despedido al efectuar un reclamo ante la empresa ${ }^{68}$. Asimismo, el Informe concluye en que los resultados adversos podrían indicar que la fórmula de igualdad de remuneraciones por un "mismo trabajo" no da cuenta de la realidad de la segregación laboral.

Los diversos estudios son coincidentes en las críticas a esta ley por su falta de eficacia ${ }^{69}$, sin perjuicio de manifestar que las carencias de esta ley han visibilizado "la situación de discriminación salarial existente y explicita el derecho a no discriminación de género".

Además, una clara evidencia de esta ineficacia de la Ley $\mathrm{N}^{0} 20.348$ es que pasados pocos años se han presentado nuevos proyectos de ley pertinentes a la materia ${ }^{70}$, los que plantean algunas medidas respecto del derecho de no discriminación remunerativa entre mujeres y varones, aunque es discutible que dichas propuestas sean suficientes para garantizar este derecho, toda vez que se limitan al mero reconocimiento del derecho.

\section{Los demás instrumentos jurídicos de tutela al derecho de igual remuneración}

Este derecho plantearía varias vías jurídicas para su garantía, las que se definirían en razón de la categoría laboral del mismo derecho. En el ámbito de la disciplina del Derecho del Trabajo en el sistema chileno, los instrumentos que podrían actuar al objetivo señalado serían los siguientes: (a) la fiscalización administrativa, (b) la tutela judicial, (c) la negociación colectiva formal e informal (d) la iniciativa empresarial.

${ }^{65}$ En cuanto señala que la Comisión toma nota de que el gobierno indica que la legislación chilena se refiere al principio de "el mismo trabajo", ya que el mercado laboral está fuertemente segregado por sexo y que por lo general las mujeres no desempeñan los mismos trabajos que los hombres. El gobierno añade que durante la discusión parlamentaria de la ley se manifestaron reticencias en cuanto a la expresión "trabajo de igual valor", ya que la misma no sería del todo clara para su interpretación. Por ello, se optó por la expresión “mismo trabajo" (OIT, 2012, p. 536).

66 OIT, 2012, pp. 536-537.

67 Cámara, 2013, pp. 56-60.

${ }^{68}$ Debido a que, como se señala “... la precaria situación en que se encuentra el o la trabajador(a) para denunciar a su empleador constituye un riesgo de perder su empleo o, al menos, ser considerado como conflictivo..." (Cámara, 2013, p. 57).

${ }^{69}$ Por todos, Díaz, 2015.

${ }^{70}$ Así, por todos, el proyecto pertinente a la materia que registra un mayor avance a la fecha es el presentado en el Senado en el 2014 (Boletín 9322-13). 
(a) En el caso chileno, la fiscalización administrativa del cumplimiento de las normas laborales está entregada a la Dirección del Trabajo y a las entidades que de ella dependen para el cumplimiento de esta materia; esto es, las Inspecciones del Trabajo. Sin embargo, a base de la información disponible, pareciera que la fiscalización de estas normas se ha reconducido a la actuación de la Inspección del Trabajo ante el reclamo específico ${ }^{71}$, el que, en conformidad con la interpretación efectuada por tal organismo, solo se puede plantear una vez que esté agotado el procedimiento interno de la empresa ${ }^{72}$. A la vez, el número de reclamos ha sido excesivamente bajo, como plantea el CEDAW y reitera un estudio de la misma Dirección del Trabajo ${ }^{73}$, en cuanto al 2014 solo había 21 denuncias efectivas; es decir, que corresponden a la materia y que se han efectuado los procesos e informes de fiscalización ${ }^{74}$.

(b) Respecto de la tutela judicial, hasta ahora son escasos los pronunciamientos de los tribunales acerca de igualdad de remuneraciones en razón de sexo, y las causas están en las dificultades para accionar judicialmente, debido a la exigencia del reclamo previo al interior de las empresas. Los casos en que ha habido pronunciamiento judicial están referidos a supuestos de discriminación directa por realización de igual trabajo, con o sin la misma denominación, y lo que se discute es la configuración de la discriminación en razón de la concurrencia o no de los pretendidos supuestos que permiten una remuneración desigual.

Más allá de los distintos resultados que se obtienen en estos casos judiciales ${ }^{75}$, la normativa de la Ley $N^{\circ} 20.348$ plantea un espacio particular: en las sentencias de los diversos casos judiciales la fundamentación del derecho de no discriminación por remuneración se plantea a base de las normas internacionales, constitucionales y específicas del Derecho del Trabajo en materia de no discriminación general, y la norma específica incorporada por la Ley N 20.348 está es el Art. 62 bis del CT se presenta como fundamento de los diferencias remunerativas que no configuran discriminación.

Por consiguiente, podría concluirse que la normativa referida (Ley N No 20.348), más que establecer una garantía al derecho de no discriminación remunerativa, abrió los supuestos de no configuración de tal discriminación, pues la acción de tutela ya estaba consagrada jurídicamente con las normas de la reforma procesal laboral.

(c) Por su parte, la negociación colectiva podría constituirse en instrumento de garantía del derecho de no discriminación, pero ello únicamente se plantea en términos potenciales, por las particularidades del sistema chileno de negociación colectiva. De una parte está la negociación colectiva formal, que es la realizada en conformidad al modelo jurídico laboral del Código del Trabajo, la que plantea una escasa cobertura

\footnotetext{
${ }^{71}$ Sin perjuicio de la interpretación acerca de las normas referidas efectuada por la misma Dirección del Trabajo, por todas, véase Dictamen 1187/18, de 10 de marzo de 2010.

${ }^{72} \mathrm{Si}$ bien sería posible sostener que la fiscalización de la Inspección puede ser inmediata, la interpretación efectuada por la Dirección del Trabajo parece ser otra, como planta el estudio de Estrella Díaz (2015, p. 73).

73 DíAz, 2015, p. 116.

${ }^{74}$ DíAz, 2015, p. 74.

${ }^{75}$ Los casos son de los Juzgados del Trabajo de Viña del Mar, 02.09.2013, Rol T.55-2013; de Valparaíso, 06.12.2013, Rol T.108-2013, y Primero de Santiago, 04.06.2017, Rol T.478-2017.
} 
tanto cuantitativamente -toda vez que las tasas tradicionales no han superado el $12 \%$ de la población laboral asalariada ${ }^{76}$ - como en términos cualitativos, en cuanto solo se ha referido a materias de remuneraciones como beneficios específicos. No obstante, a partir de la entrada en vigencia de la Ley $\mathrm{N}^{\circ} 20.940$, de 2016, se plantean visiones más optimistas en cuanto al potencial de este instrumento como vía de garantía ante la discriminación laboral de género, a causa de la explícita referencia a la posibilidad de que las medidas de equidad de género sean objeto de la negociación colectiva (Art. 306, inciso 2.do, CT $)^{77}$ o el acceso que tendrán las organizaciones sindicales a la información respecto de remuneraciones con ocasión del ejercicio de la negociación colectiva ${ }^{78}$.

Por otra parte, la negociación colectiva informal, que es la que se realiza al margen de las estrictas normas laborales ${ }^{79}$, también tendría el potencial para establecer este instrumento de garantía. Precisamente, la manifestación más acabada de garantía de igual remuneración por trabajo de igual valor que se ha logrado hasta ahora es fruto del acuerdo entre la Asociación Nacional de Empleados Fiscales (ANEF) y el gobierno, de julio de 2015, referido a la "evaluación de los puestos de trabajo con perspectiva de género, para la disminución de brechas salariales en el Estado" ${ }^{80}$ y que se implementó en una repartición, el Instituto de Salud Laboral (ISL) ${ }^{81}$.

(d) La iniciativa empresarial. Cierto es que, además de los instrumentos de origen estatal y de los que provienen de la autonomía colectiva, está la propia iniciativa de las empresas para obligarse en materia de resguardo de la igualdad de remuneraciones.

La vía institucional hasta ahora establecida es la certificación en Norma ISO respecto de la materia, que corresponde a la Norma Chilena (NCh) 3262-2012, referida a un Sistema de Gestión de Igualdad de género y conciliación de la vida laboral, familiar y personal, la que establece normas de igualdad de compensaciones. La certificación de las empresas es voluntaria y hasta le fecha, noviembre de 2019, se han registrado 14 entidades $^{82}$ en el Servicio Nacional de la Mujer y de Equidad de Género (SERNAMEG) ${ }^{83}$.

De otra parte, existen otras posibilidades, como es la Iniciativa de Paridad de Género (IPG), la que, de acuerdo con su definición, constituye una "alianza público-privada promovida por el Banco Interamericano de Desarrollo y el Foro Económico Mundial, que tiene como propósito reducir las brechas de género y aumentar la participación económica y el progreso de las mujeres en el mercado laboral en nuestro país" 84 .

76 Aunque por un cambio de medición se ha planteado un aumento que la OCDE cifra en 18,9\%, igualmente se objetan estas cifras por un cambio de la metodología que no daría cuenta de la real cobertura. Al respecto, véase Durán y Gamonal, 2019, pp. 7-9.

77 CaAmaño, 2018, pp. 63-70.

78 GÁlvez y Díaz, 2017, p. 45.

79 RoJAs, 2014, pp. 125-129.

${ }^{80}$ Gobierno de Chile, ANEF y OIT, 2018, p. 64.

${ }^{81}$ Bentancor y UReta, 2018, p. 38.

82 De acuerdo con correo del pasado 29 de noviembre de 2019, emitido por el SERNAMEG, por información solicitada por medio de Ley de Acceso a la Información Pública.

83 Véase nota 49.

${ }^{84}$ IPG: 2019a. 
Al efecto, dicha iniciativa ha dispuesto 10 medidas y una de ellas está referida a la Medición y corrección de brechas salariales de género en el sector público y privado ${ }^{85}$. Si bien a la fecha se indica que hay 170 empresas adheridas, no se tienen estudios a disposición que den cuenta de las medidas concretas dispuestas en estas empresas ante la brecha salarial.

\section{CONCLuSiones}

De acuerdo con los imperativos de las normas internacionales, en este trabajo se han planteado los objetivos de identificar los instrumentos jurídicos existentes en el sistema chileno que permitan garantizar el derecho de no discriminación remunerativa entre mujeres y hombres, como también analizar la eficacia de los mismos. A continuación se presentan las conclusiones o, más bien, las constataciones que arroja este trabajo.

En primer término, se destaca el desconocimiento del contenido del derecho fundamental de no discriminación remunerativa entre mujeres y hombres, y que es el de igual remuneración por trabajo de igual valor, y que se manifiesta tanto en la comunidad jurídica como política, ignorando por consiguiente múltiples manifestaciones de la discriminación remunerativa, tanto directa como indirecta. Y ello en circunstancias de que el Estado de Chile está vinculado por tratados que ha ratificado, por los que no solo tiene la obligación de reconocer este derecho en la dimensión indicada, sino que también está obligado a su promoción, como lo establecen el Convenio 100 de OIT y la CEDAW de la ONU.

En segundo término, los instrumentos para tutelar el derecho de no discriminación remunerativa han sido ineficaces en el objetivo de garantizar el derecho de no discriminación remunerativa, inclusive en la dimensión limitada al "trabajo igual”.

En efecto, el instrumento de la obligación general del empleador de "dar cumplimiento al principio de igual remuneración por igual trabajo", que fue establecido por la Ley $\mathrm{N}^{\mathrm{0}} 20.348$, de 2009, es insuficiente en cuanto solo reiteró una obligación que ya establecía el ordenamiento jurídico, debido a la eficacia inmediata de los derechos fundamentales en el ámbito de las relaciones laborales; y su único instrumento de promoción, el registro de puestos de trabajo, lo circunscribió a la gran empresa.

Además, este instrumento limitó los de carácter general, como son el reclamo administrativo y la acción judicial: de una parte, por la adición especial de supuestos que no configurarían la discriminación y, de la otra, requiriendo el reclamo previo al interior de la empresa, inhibiendo, por consiguiente, dichas gestiones.

Es cierto que el origen de estas limitaciones que se impusieron al instrumento de la Ley N $N^{\circ} 20.348$ fue la Comisión del Trabajo del Senado, cuya discusión y argumentación requiere un examen por la falta de consideración de los antecedentes del sistema de relaciones laborales, tanto del chileno como de los comparados, y ello más allá del

${ }^{85}$ IPG, 2019b. 
necesario análisis político con el fin de explicar los acuerdos que desconocen dimensiones esenciales del derecho en cuestión, específicamente del trabajo de igual valor.

Otros instrumentos son los de carácter general que operan en el ámbito del ordenamiento jurídico laboral. Sin embargo, ellos tienen escasa posibilidad de actuar como mecanismo de promoción y de garantía del derecho de no discriminación laboral. En efecto, el reclamo administrativo y la acción judicial están supeditados al reclamo previo al interior de las empresas y, debe observarse que, aunque no estuviesen condicionados estos mecanismos son insuficientes. Asimismo, la negociación colectiva en Chile está escasamente desarrollada, por lo que en su estado actual impide constituir una vía de garantía de este derecho. No obstante, sí existe una experiencia de negociación colectiva informal en la que se implementó un mecanismo de tutela, mediante la valoración de los puestos de trabajo sin sesgo de género; empero esta fue una experiencia aislada, en un contexto especial que la promovió. Por lo demás, las vías de iniciativa empresarial, tanto la NCh 3262-2012 como la Iniciativa de Paridad de Género, por muy loables que sean, tienen un alcance limitado que no es apto para garantizar un derecho fundamental, así como es el de no discriminación remunerativa.

En tercer término, una dimensión que atraviesa el análisis de las garantías para el derecho de no discriminación retributiva es la del sistema de relaciones laborales, tanto en relación con cualquier sistema jurídico como respecto del chileno. Más allá de las particularidades de nuestro sistema que han sido explicitadas, como es la escasa negociación colectiva, hay otras como es la desregulación de la gestión de las empresas en materia laboral y la falta de participación de la organización de los trabajadores en dichas materias, sujetando dicha organización a lo que decida la dirección de cada empresa, como es, a vía de ejemplo, la falta de clasificación empresarial y de los puestos de trabajo, en circunstancias de que ello está definido en diversos sistemas e, inclusive, constituye materia de negociación colectiva.

Ciertamente que el establecimiento de diversos instrumentos de garantía del derecho de no discriminación remunerativa pasan o pueden pasar por lo que es la organización de la gestión laboral y, por consiguiente, cualquier medida que se establezca deberá considerar y modificar el sistema de gestión laboral existente hasta ahora.

Asimismo, debe reiterarse la constatación efectuada de la casi inexistencia de instrumentos jurídicos que garanticen el derecho de no discriminación remunerativa en nuestro sistema jurídico. Es cierto que el sistema de relaciones laborales chileno conoce el problema de la brecha salarial, pero la labor de construir un sistema de garantías a este derecho está en sus inicios. Por último, falta reflexión como también consideración de los sistemas comparados ${ }^{86}$ que en los últimos decenios y, particularmente, en los últimos años elaboran y revisan los instrumentos para la garantía de una real igualdad de mujeres y varones, en especial en el mundo del trabajo.

${ }^{86}$ Véanse los señalados en nota 30. 


\section{BiBLIOGRAFÍA}

Ballester, Amparo: 2018a, La discriminación retributiva por razón de sexo, Albacete: Bomarzo.

Ballester, Amparo: 2018 b, "La discriminación retributiva por razón de sexo en la Unión Europea”, Revista Documentación Laboral N. 115, Volumen 3.

Barrere, Ángeles: 1996, Discriminación, Derecho Antidiscriminatorio y Acción Positiva en favor de las Mujeres", Madrid: Civitas.

Bentancor, Andrea y Ureta, Camila: 2018, Promoviendo el Diálogo Social en Chile: Evaluación de los puestos de trabajo con perspectiva de género, para la disminución de brechas salariales en el Estado, Dirección de Presupuestos, Ministerio de Hacienda.

Biblioteca del Congreso Nacional, BCN: 2009, Historia de la Ley $N^{\circ} 20.348$. Disponible en: file://C:/Users/Usuario/Downloads/Historia\%20de\%20la\%20Ley\%2020348.pdf (fecha de consulta: 28 de marzo de 2018).

Brega, Carla; Durán, Gonzalo y Sáez, Benjamín: 2015, "Mujeres trabajando. Una exploración al valor del trabajo y la calidad del empleo en Chile", Fundación Sol. Disponible en: http:// www.fundacionsol.cl/wp-content/uploads/2015/03/Estudio-Mujeres-Trabajando-2015.pdf (fecha de consulta: 28 de marzo de 2018).

CAAMAÑo, Eduardo: 2018, "La negociación colectiva como vía para promoción de la conciliación de trabajo y vida familiar", en Arellano, Pablo (editor) La Discriminación en la legislación social chilena, Valparaíso: Ediciones Universitarias de Valparaíso.

CAAmaño, Eduardo: 2003, "La tutela al derecho de no discriminación por razón de sexo durante la vigencia de la relación laboral, Revista de Derecho (Valdivia), Volumen XIV, No 1.

CaAmaño, Eduardo: 2001, "La discriminación laboral indirecta", Revista de Derecho (Valdivia), Volumen XII.

Cabezas, Jaime: 2012: "La discriminación retributiva por razón de sexo como paradigma de discriminación sistémica”, Lan Harremanak, Revista de Relaciones Laborales de la Universidad del País Vasco, $\mathrm{N}^{\circ} 25$.

CÁmara de Diputados: 2013, Departamento de la Evaluación de la Ley, "Evaluación de la Ley $\mathrm{N}^{\circ}$ 20.348. Resguarda el derecho a la igualdad de remuneraciones", junio de 2013, 64 páginas. Disponible en http://www.evaluaciondelaley.cl/foro_ciudadano/site/artic/20130325/asocfile/20130325153119/informe_ley_nro_20348.pdf (fecha de consulta: 6 de julio de 2018).

CEDAW, Comité para la Eliminación de la Discriminación contra la Mujer: 2012, Informe "Observaciones finales sobre los informes periódicos quinto y sexto de Chile". Disponible en: file://C:/Users/Usuario/Desktop/Proyecto\%202019/Informes\%20Internacionales/CEDAW/ CEDAW.OBSERVACIONES_FINALES_COMITE_5y6_-INF.pdf (fecha de consulta: 11 de julio de 2019).

CHICHA, Marie-Therese y OIT: 2006, “A comparative analysis of promoting pay equaty: models and Impacts”, Ginebra, Oficina Internacional del Trabajo.

COMUnidad Mujer: 2015. "Mujer y trabajo: Brecha salarial, la gran deuda con las trabajadoras en Chile", Serie n. 32.

Consejo de Europa, 1961, Carta Social Europea.

DíAz, Estrella: 2015, La desigualdad salarial entre hombres y mujeres. Alcances y limitaciones de la Ley $N^{\circ} 20.348$ para avanzar en justicia de género, Santiago, Dirección del Trabajo.

DíAz, Iván: 2013, "Ley chilena contra la discriminación. Una evaluación desde los derechos internacional y constitucional”, Revista Chilena de Derecho, Volumen $40 \mathrm{~N}^{\circ} 2$.

DurÁN, Gonzalo y Gamonal, Sergio: 2019, "La opacidad de las cifras: la cobertura de negociación colectiva en Chile, aceptada en Revista Derecho y Crítica Social. Disponible en: https:// derechoycriticasocial.com/about/informacion-general/ 
GÁLVEz, Thelma y Díaz, Estrella: 2017, Mismo pago por trabajo de igual valor: Avance efectivo hacia la superación de la brecha salarial entre mujeres y hombres, Santiago, Dirección del Trabajo, Departamentos de Estudios, Dirección del Trabajo.

GobIERno de Chile, ANEF y OIT, 2018. Memoria de los puestos de trabajo con perspectiva de Género, para la disminución de brechas salariales en el Estado”, marzo de 2018, 64 páginas.

INE: 2019, Encuesta Suplementaria de Ingresos, ESI, 2018, p. 4. Disponible en: https://www.ine.cl/docs/ default-source/encuesta-suplementaria-de-ingresos/publicaciones-y-anuarios/s\%C3\% ADntesisde-resultados/2018/sintesis_nacional_esi_2018.pdf?sfvrsn=eed2fa51_3 (fecha de consulta: 2 de noviembre de 2019).

IPG: 2019a, Iniciativa de Paridad de Género. Disponible en: https://iniciativaparidadgenero.cl/ (fecha de consulta: 21 de noviembre de 2019).

IPG: 2019b, Iniciativa de Paridad de Género, "Diez Medidas". Disponible en: https://iniciativaparidadgenero.cl/10-medidas/ (fecha de consulta: 21 de noviembre de 2019).

Lousada, José: 2014, El derecho fundamental a la igualdad efectiva de mujeres y hombres. Valencia: Tirant lo Blanch.

Lousada, José; Cabezas, Jaime y Núñez-Cortez, Pilar: 2018, Curso de Igualdad y Diversidad en las Relaciones Laborales, Valencia, Tirant lo Blanch.

MuÑoz, Fernando: 2018, "La necesidad de una interpretación pro discriminado de la acción contra la discriminación, Revista de Derecho (Valdivia), Volumen 31, No 2.

OIT: 2019, Informe Mundial sobre Salarios 2018/19. ¿Qué hay detrás de la brecha salarial de género?, Ginebra, Oficina Internacional del Trabajo.

OIT: 2013, Igualdad Salarial. Guía Introductoria, Ginebra, Oficina Internacional del Trabajo.

OIT: 2012. "Informe de la Comisión de Expertos en Aplicación de Convenios y Recomendaciones Conferencia Internacional del Trabajo, 101ª . reunión, 2012”, Informaciones y memorias sobre la aplicación de convenios y recomendaciones. Disponible en: https://www.ilo.org/wcmsp5/groups/ public/---ed_norm/---relconf/documents/meetingdocument/wcms_174831.pdf (fecha de consulta: 11 de julio de 2019).

OIT: 2008, Promoción de la Igualdad Salarial por medio de la evaluación no sexista de los empleos, Ginebra, Oficina Internacional del Trabajo,

RojAs, Irene: 2014, "La negociación colectiva en Chile: La legal y la general”, en Rojas Miño, Irene (coordinadora), La negociación colectiva en Chile, Santiago: Librotecnia.

SÁEz, Carmen: 1994, Mujeres y Mercado del Trabajo. Las discriminaciones directas e indirectas, Madrid: Consejo Económico y Social.

Unión Europea: 2006, Directiva Comunitaria 2006/54, Diario Oficial de la Unión Europea, 26.7.2006.

Unión Europea: 2014, Recomendación de la Comisión de 7 de marzo de 2014. Diario Oficial de la Unión Europea, 8.3.2014.

\section{Normas jurídicas citadas}

ONU, Declaración Universal sobre Derechos Humanos, adoptada con fecha 10 de diciembre de 1948.

OIT, Convenio 100, sobre Igualdad de Remuneraciones, adoptado con fecha 29 de junio de 1951. Publicado en Chile el 20 de septiembre de 1971.

OIT, Convenio 111, sobre Discriminación de empleo y ocupación, adoptado con fecha 25 de junio de 1958. Publicado en Chile el 20 de septiembre de 1971.

CEDAW, Convención sobre Eliminación de Todas las Formas de Discriminación contra la Mujer, adoptada el 18 de diciembre de 1979. Publicada en Chile el 9 de diciembre de 1989. 
LEy N 19.759 que modifica el Código del Trabajo, publicada con fecha 8 de noviembre de 1998.

LEy No 19.591 , Modifica el Código del trabajo en materia de protección a la maternidad, publicada con fecha 8 de noviembre de 1998.

CT, Código del Trabajo, 2003, D.F.L. No 1, "Fija el texto refundido coordinado y sistematizado del Código del Trabajo".

OIT, Conferencia Internacional del Trabajo, 2004, Resolución relativa a la promoción de la igualdad entre hombres y mujeres, a la igualdad de la remuneración y a la protección de la maternidad, Disponible en https://www.ilo.org/wcmsp5/groups/public/---dgreports/--gender/documents/meetingdocument/wcms_100570.pdf (fecha de consulta: 19 de noviembre de 2019).

LEy N $\mathrm{N}^{\circ} 20.260$, modifica el Libro V del Código del Trabajo, publicada el 29 de marzo de 2008.

LEY $\mathrm{N}^{\circ} 20.348$, que resguarda el derecho a la igualdad de las remuneraciones, publicada el 19 de junio de 2009.

LEY $\mathrm{N}^{\circ} 20.416$ que fija normas especiales para empresas de menor tamaño, publicada el 3 de febrero de 2010.

Dictamen Dirección del Trabajo, 1187/18, sobre igualdad de remuneraciones, de 10 de marzo de 2010.

Proyecto DE LEY que establece medidas en materia de discriminación e igualdad de remuneraciones de hombres y mujeres. Boletín 9322-13.

\section{Jurisprudencia citada}

Primer Juzgado del Trabajo de Santiago, sentencia de 4 de junio de 2017, T.478-2017. JuZgado del Trabajo de ViÑa del MAR, sentencia de fecha 02 de septiembre de 2013, T.55-2013. Juzgado del Trabajo de Valparaíso, sentencia de fecha 6 de diciembre de 2013, T-108-2013. Primer Juzgado del Trabajo de Santiago, sentencia de 2 de diciembre de 2019, T-57-2009. 
